News

\title{
Delay aging of the brain cells and improve memory without medications
}

Volume 4 Issue I - 2015

Mohamed M Hassan

President at Dr. Hassan Chiropractic \& Natural healing, USA

Correspondence: Mohamed M Hassan, President at Dr. Hassan Chiropractic \& Natural healing, Texas Chiropractic College, USA, Email dr.mmhassan@yahoo.com

Received: October 16,2015 | Published: October 19, 2015 acid regained so much of their Vigor and youth (Neuro Chemical Research at University of California).

\section{Aging and the free radical theory}

I. Scientists studying aging found that our Cells and the DNA which contains our genetic code Sustain damage by highly reactive chemicals referred to as 'Free Radicals'.

II. -Free radicals are the Result of Normal Metabolic processes, or as the result of nutritional deficiency, these damaging chemical species create havoc with our cells and cause us to age.

III. Mitochondria (cell power house) generate energy inside each cell by burning food in the presence of oxygen. Subjecting Mitochondria to high levels of free radical damage.

IV. -As we age, Mitochondria become so damaged by Free Radicals as they lose their ability to Function efficiently due to diminishing Cellular Energy and we become subject to degenerative diseases including but not limited to memory loss or Alzheimer disease .

V. Researchers found that supplementation with R-alpha Lipoic Acid plus Acetyl L-Carnitine (LA/ALC) in rats, significantly lower levels of naturally present antioxidants and Superoxide Dismutase (SOD) activity (free radicals).

VI. Feeding LA/ALC to the old rats partially restored age-associated mitochondrial dysfunction to the levels had by the young rats.

VII. - These results document that mitochondria decay plays an important role in brain aging.

VIII. Combination of nutrients targeting mitochondria can ameliorate mitochondrial decay by preventing oxidative damage and delay aging.

IX. Researchers Recommend LA/ALC for Prevention of Parkinson's disease.

\section{Other factors which may help improve} memory and delay aging brain

i. Avoid Aspartaine (sweetener) -- used in beverages or commercial food.

ii. As Aspartaine -- biochemical end product -- is Formaldehydes, which they are very damaging to memory and aging brain -(Avoid "soft" (carbonated, sweetened) beverages, canned goods, alcoholic beverages, artificial sweeteners or products containing these ingredients.

iii. Exercise is important to improve memory --- by improving the general blood circulation --- you can do simple exercise to improve memory --like: jumping the robes, or walking backward for short distance at beginning --then increase the distance gradually --these simple exercises will help improve the memory.

iv. Get enough sleep, Avoid stress.

v. Be nutritionally sound: Eat more vegetable, less meat (turkey, chicken, pork, caw meat).

vi. It is good to eat lamb meat (high in Omega 3 and Carnitine) -- [Omega 3 are good anti inflammatory, and Carnitine -- are good fuel for the heart and brain] -- which help delay aging and improve memory.

vii. Be hormonally sound/balanced: Nutritional hormonal support: eat soy, fish oil, Sardines, gingko, and take vitamin B-complex help balance your hormones to maintain memory and delay aging.

viii. **Diet concept that may help memory and delay aging brain.

\section{Basic macrobiotic dietary concepts}

\section{The following are basic concepts of the macrobiotic way of eating}

I. Consume organically and locally-grown foods in season as they become available, or foods that will store without artificial preservation or refrigeration. Alternatively, eat foods grown in the same latitude.

II. Consume cooked whole grains primarily, and recipes made from whole grains; secondarily in quantity, cooked vegetables, adjusting proportions and preparation methods according to activity, climate and seasonal fluctuations of temperature and humidity. 
III. Use solar-evaporated Sea Water Salts in preparation of foods, as well as traditionally-aged miso and shoyu, umeboshi and seaweeds.

IV. Drink un dyed, 3-year old roasted twig tea (kukicha) as preferred beverage.

V. Use unrefined, Cold-Pressed Seed Oils sparingly, made from organically-grown seeds, like sesame, corn, safflower, sunflower, flaxseed, etc.

VI. Use roasted seeds and nuts, fruit, salads and fish occasionally as desired, in smaller quantities, as provided in season.

VII. Use beans and bean products frequently, as primary sources of protein, along with whole grain and vegetable dishes.

VIII. Avoid all foods and beverages containing refined sweeteners, chemical dyes, synthetic flavorings or seasonings, refined oils, chemical preservatives, or made from foods grown with chemical insecticides, herbicides, fungicides, chemical fertilizers, or produced by bio-engineering or grown under hot-house conditions.
IX. Avoid foods grown in, and shipped from, Warmer latitudes.

X. Avoid "soft" (carbonated, sweetened) beverages, canned goods, alcoholic beverages, artificial sweeteners or products containing these ingredients.

XI. Chew each mouthful thoroughly before swallowing.

\section{Acknowledgments}

None.

\section{Conflicts of interest}

Author declares there are no conflicts of interest.

\section{Funding}

None. 